\title{
Proyecto ULPGC-UniZambeze. Formación médica universitaria en países en desarrolllo
}

\author{
Luis López-Rivero, Rosario Berriel-Martínez, Gloria González-Azpeitia, José Ángel García-Hernández, \\ Felipe Rodríguez de Castro
}

\begin{abstract}
Resumen. Todos los países, cualquiera que sea su grado de desarrollo, se esfuerzan por proporcionar unos cuidados sanitarios adecuados a sus ciudadanos. Gozar de buena salud es una condición básica para el desarrollo y la seguridad de las personas, a pesar de lo cual persisten enormes desigualdades en salud entre los países. Los educadores médicos tienen un papel clave en la reducción de estas desigualdades al abordar uno de los aspectos esenciales del problema: la acusada escasez de médicos que sufren algunas regiones, especialmente en el África subsahariana. Una de las mayores dificultades para afrontar esta cuestión es la falta de profesores cualificados, lo que podría solventarse mediante estancias cortas de graduados procedentes de países desarrollados que voluntariamente quisieran participar en este tipo de iniciativas. Pero el mundo de la educación médica tampoco es un terreno justo, equilibrado y equitativo, y trabajar en países pobres y alejados supone un reto importante. En este artículo, presentamos la experiencia en el diseño y desarrollo de un proyecto de formación de médicos en la Universidad de Zambeze, en Tete (Mozambique), Ilevada a cabo desde la Universidad de Las Palmas de Gran Canaria.
\end{abstract}

Palabras clave. Educación médica. Formación de médicos. Mozambique. Países en desarrollo.

\section{ULPGC-UniZambeze project. Undergraduate medical education in developing countries}

Summary. Every nation -rich or poor and developed or developing- strives hard to deliver optimal healthcare to its citizens. Good health is the necessary condition for development and human security, but inequities in health persist. Medical educators have a key role to play in reducing inequities in global health by addressing the underlying doctor shortages that have reached crisis levels in some regions. The region in which these health inequities and doctor deficiencies are starkest is sub-Saharan Africa. In poor countries, a major constraint to approach this issue is the scarcity of qualified teachers. One of the options is the short-term placement of graduates from rich countries seeking opportunities to contribute in other countries that are severely deficient in faculty. But the world of medical education is not a level playing field and working in developing countries, and in remote areas in particular, can be challenging. In this article we show a recent experience carried out at the University of Las Palmas de Gran Canaria designing and supporting a project on undergraduate medical education at the University of Zambeze, in Tete, Mozambique.

Key words. Developing countries. Medical education. Mozambique. Undergraduate medical education.

\section{Introducción}

Gozar de buena salud es la condición necesaria -si bien no suficiente- para el desarrollo humano. Sin embargo, las desigualdades en salud aún persisten, tanto entre países como dentro de una misma región. La insuficiencia de médicos está en el trasfondo de este problema y ha alcanzado niveles críticos en algunas zonas del mundo. Desde este punto de vista, el papel potencial de los educadores médicos en la reducción de estas desigualdades es clave. Posiblemente, la región del mundo en la que las desigualdades en salud se hacen más evidentes y la ca- rencia de médicos resulta más patente es el África subsahariana [1].

En los países pobres, una de las mayores limitaciones que plantea el abordaje del déficit de médicos es la escasez de profesores cualificados [2]. Entre las opciones que se han planteado para acometer este obstáculo está el desplazamiento, por períodos cortos, de facultativos que desean cooperar en países en vías de desarrollo con un déficit grave de médicos [3]. Tales actividades, sin embargo, deben estar enmarcadas en estrategias más amplias orientadas a fortalecer la capacitación de los países más pobres [4]. En este sentido, las iniciativas lleva-
Facultad de Ciencias de la Salud. Universidad de Las Palmas de Gran Canaria. Las Palmas de Gran Canaria, España.

Correspondencia:

Dr. Felipe Rodríguez de Castro. Facultad de Ciencias de la Salud. Universidad de Las Palmas de Gran Canaria. Paseo Blas Cabrera Felipe, s/n. E-35016 Las Palmas de Gran Canaria.

E-mail:

frodcasw@ulpgc.es

Profesores que han

participado en el proyecto:

J. Alonso Bilbao, B. Alonso Ortiz,

R. Amela Peris, J. Armas Molina,

R. Bautista Salinas, A. Benito Reyes,

A. Blanco Díez, J.M. Bosch Benítez,

F. Cabrera López, L.M. Calvo Hernández,

P. Camargo Camacho, C. Castro Díez,

M. Cazorla Betancor, C. Delgado

Godoy, R. Díaz Morán, E. Dolz Bubi,

D. Durán Moreno, T. Fernández

González, J. Freixinet Gilart, J.A.

García Hernández, S. García Mendieta,

R. González Aguado, G. González

Azpeitia, G. Guerra Jiménez,

L. Gutiérrez García, M. Hathiramani Sánchez, Y. Hernández Gago,

L. Hernández González, N. Jaén Sánchez, M.J. López-Madrazo Hernández, L.M. López Rivero, A. Marcos García, A. Martín Martínez, J. Medina Henríquez, F. Melián Nuez, C. Mendoza Guzmán, T. Molero Labarta, D. Montesdeoca Cabrera, G. Muratore Moreno, J. Navarro Esteva, J. Ortega Trujillo, D. Pérez Alonso, N. Pérez Martín, C. Pérez Mato, F. Rodríguez de Castro, R. Sánchez Hernández, L. Santana Ortega, S. Suria González, A. Sierra Hernández, O. Sobral Caraballo,

E. Soler Cruz, A. Tugores Cester, A. Wagner Fahlin.

Agradecimientos:

M. Guerra Medina, técnico del CUCID.

\section{Recibido:}

07.10.16.

Aceptado:

13.10.16.

Conflicto de intereses:

No declarado. 
das a cabo desde Asia, Canadá y Europa con algunos países de Oriente Medio y África proporcionan evidencias claras de que la educación médica libre de prejuicios nacionales, es decir, 'globalmente neutral', es aceptada más rápidamente como una herramienta potencial de desarrollo para estimular y enriquecer la calidad de la enseñanza y, consecuentemente, el sistema de salud de un país.

En este artículo se muestra una experiencia reciente de la Universidad de Las Palmas de Gran Canaria (ULPGC) en el diseño y ejecución de un título de graduado en medicina en la Universidad de Zambeze (UniZambeze), en Mozambique.

\section{Origen del proyecto}

El proyecto de cooperación UniZambeze-ULPGC se gestó en octubre de 2010, en el transcurso del III Encuentro Internacional de Universidades con África, celebrado en Las Palmas de Gran Canaria. En aquella reunión, representantes de la institución mozambiqueña manifestaron la apremiante necesidad de contar con personal docente cualificado para asegurar la formación clínica de los estudiantes de medicina de aquella universidad. La formación de médicos en la UniZambeze había comenzado en 2009 contando con profesorado cubano y mozambiqueño cualificado para impartir los cursos preclínicos de la titulación. Sin embargo, no se disponía de docentes en los ámbitos de conocimiento de medicina, cirugía, pediatría y obstetricia-ginecología, por lo que de no encontrar una rápida solución para cubrir esa carencia, los responsables de la UniZambeze se verían abocados a suspender esos estudios. Desde la Facultad de Ciencias de la Salud de la ULPGC se planteó como una opción viable la posibilidad de que sus profesores colaboraran con la UniZambeze en la solución de este problema.

\section{Marco geográfico y social}

La UniZambeze es una universidad pública de Mozambique, fundada en marzo de 2008, que oferta estudios de enseñanza superior para los habitantes de la región central del país, conocida como Zambezia (Fig. 1). La sede rectoral se encuentra en la ciudad de Beira, donde se imparten la mayoría de las titulaciones, mientras que la Facultad de Ciencias de la Salud se ubica en la ciudad de Tete, en la provincia del mismo nombre. Además de medicina, en el campus de Tete también se ofertan los grados de odontología y de farmacia.
Mozambique se encuentra entre los diez países peor situados en la clasificación mundial del índice de desarrollo humano del Programa de las $\mathrm{Na}$ ciones Unidas para el Desarrollo. En concreto, en la provincia de Tete, la esperanza media de vida es de 55 años y la tasa de médico por habitante, de 1 por 50.000, lo que extrapolado a nuestro país supone que se dispondría de unos 800 médicos para toda la población española. Antes de comenzar el actual proyecto, en Tete, con una población cercana a los dos millones de habitantes, se contaba oficialmente con un cirujano, un ginecólogo, dos internistas y dos pediatras. A este reducido número de médicos hay que añadir otros problemas objetivos que dificultan la prestación sanitaria y el acceso a la salud de esta población, como son la extensión del territorio (superior a Castilla-León) y unas vías de comunicación terrestre muy deficientes. Con esta información parecía evidente la necesidad perentoria de incrementar el número de médicos en la región y, por consiguiente, quedaba fuera de toda duda la pertinencia del proyecto que se planteaba.

Desde el punto de vista de las infraestructuras, la Facultad de Ciencias de la Salud de la UniZambeze ha contado desde su inicio con fondos de la cooperación norcoreana, lo que ha posibilitado que a partir del año 2012 se pudiera disponer de unos espacios específicos para la docencia teórica de las tres titulaciones y de material ofimático y de proyección de imágenes adecuado (Fig. 2). Actualmente, una de las principales carencias es la falta de una biblioteca con un fondo bibliográfico mínimo aceptable. El Hospital Provincial de Tete es un hospital general que presta atención sanitaria en las especialidades médicas y quirúrgicas básicas. Tiene una dotación de 300 camas distribuidas en pabellones temáticos, dispone de un laboratorio general y microbiológico básico y tiene capacidad para realizar radiografías simples y ecografías. Las carencias en recursos materiales, técnicos y humanos son evidentes, pero existe un elevado nivel organizativo que permite la realización de prácticas clínicas.

\section{Desarrollo del proyecto}

Una vez aprobada por el rectorado la participación del profesorado de la ULPGC en el proyecto de cooperación, se designó al profesor Luis López Rivero como director de éste, con el encargo de diseñar el plan de estudios y coordinar su ejecución. Durante 2011 se perfiló el plan docente del segundo ciclo del 
Figura 1. Regiones de Mozambique.

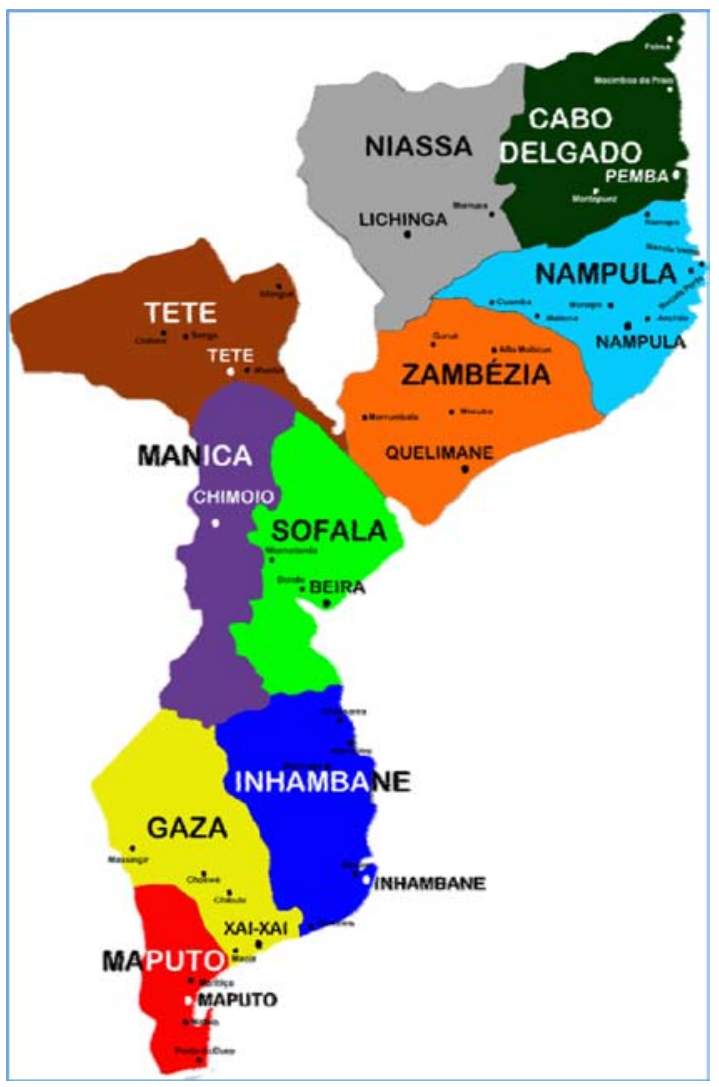

grado de medicina de la UniZambeze, con la participación del decano y de la vicerrectora de Ordenación Académica de la universidad mozambiqueña. Las materias específicas, su extensión, contenidos y objetivos de aprendizaje se delinearon de acuerdo a la realidad sanitaria del país (Tabla) y utilizando como referencia obligada los programas de la Facultad de Medicina de la Universidad Eduardo Mondlane, en Maputo.

Ante la imposibilidad de disponer de un número suficiente de profesores universitarios $-o$ incluso de especialistas con experiencia docente- que pudieran abandonar sus proyectos profesionales y personales para residir durante el curso lectivo completo en la ciudad de Tete, se diseñó un plan docente 'intensivo y modular', ya utilizado en algunas universidades africanas con las que colaboran otras instituciones occidentales. En base a este plan, cada asignatura se imparte, tanto la teoría como la práctica, de forma exclusiva durante un período de
Figura 2. Imagen de una de las aulas de la Facultad de Ciencias de la Salud de la Universidad de Zambeze.

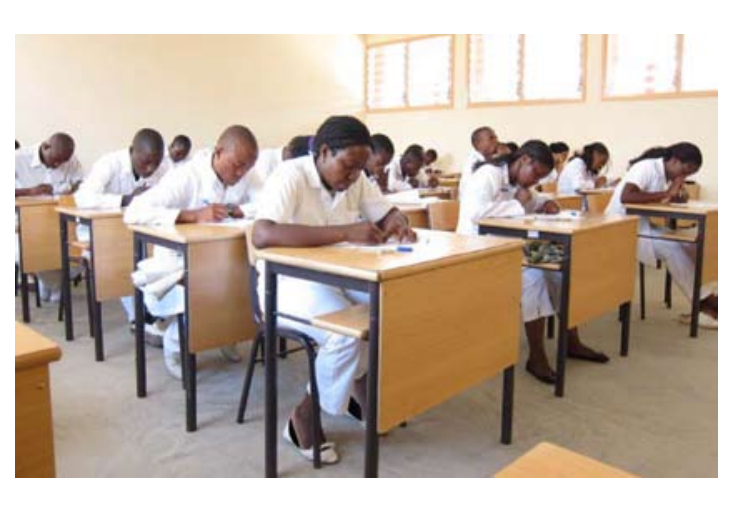

cuatro a seis semanas. Con esta planificación de las estancias, un número suficiente de profesores de nuestra facultad se ofrecieron a participar de forma totalmente altruista, sin retribución económica alguna y, en la mayoría de las ocasiones, dedicando su período vacacional para impartir las clases en Tete.

En febrero de 2012, la ULPGC y la UniZambeze firmaron un convenio específico de colaboración. El objetivo fundamental de este convenio fue fortalecer las capacidades institucionales y de formación de la Facultad de Ciencias de la Salud de la UniZambeze a través de la participación de profesores de la ULPGC y de otras instituciones colaboradoras en la enseñanza de materias relacionadas con las áreas de conocimiento de medicina, cirugía, terapéutica, pediatría y obstetricia- ginecología de los cursos cuarto, quinto y sexto del grado de medicina. De esta forma se contribuía a dotar a los futuros graduados de los conocimientos, habilidades y herramientas metodológicas necesarias para ejercer como médicos en su país.

Desde febrero de 2012 hasta noviembre de 2015, un total de 45 profesores de la ULPGC se han desplazado en distintas ocasiones a Tete, donde han permanecido por períodos de tres a cuatro semanas como media. La universidad canaria se encarga del transporte y del seguro de los docentes, mientras que la mozambiqueña cubre los gastos de alojamiento y manutención de los profesores durante su estancia en el país. El traslado de los docentes se gestiona desde el Vicerrectorado de Internacionalización y Cooperación de la ULPGC, a través del Centro Universitario para la Cooperación Internacional al Desarrollo (CUCID). 
Tabla. Plan de estudios de cuarto, quinto y sexto curso del Grado de Medicina de la Universidad de Zambeze y distribución de créditos ECTS (European Credit Transfer and Accumulation System).

\begin{tabular}{|c|c|c|}
\hline & & ECTS \\
\hline \multirow{4}{*}{$\begin{array}{l}\text { 4.ㅇ curso: } \\
\text { primer semestre }\end{array}$} & Medicina Interna I (digestivo, neumología, cardiología y hematología) & 12 \\
\hline & Cirugía I (general, torácica y vascular) & 8 \\
\hline & Terapéutica I & 5 \\
\hline & Salud Comunitaria V & 5 \\
\hline \multirow{4}{*}{$\begin{array}{l}\text { 4. }{ }^{\circ} \text { curso: } \\
\text { segundo semestre }\end{array}$} & Medicina Interna II (nefrología, reumatología, endocrinología y neurología) & 12 \\
\hline & Cirugía II (abdominal y urología) & 8 \\
\hline & Terapéutica II & 5 \\
\hline & Salud Comunitaria VI & 5 \\
\hline \multirow{5}{*}{$\begin{array}{l}\text { 5. }{ }^{\circ} \text { curso: } \\
\text { primer semestre }\end{array}$} & Medicina Interna III (dermatología y enfermedades de transmisión sexual) & 6 \\
\hline & Cirugía III (ortopedia y traumatología) & 6 \\
\hline & Ginecología y Obstetricia I & 7 \\
\hline & Pediatría I & 7 \\
\hline & Salud Comunitaria VII & 4 \\
\hline \multirow{6}{*}{$\begin{array}{l}\text { 5. - curso: } \\
\text { segundo semestre }\end{array}$} & Medicina Interna IV (psiquiatría, cuidados intensivos y reanimación) & 6 \\
\hline & Cirugía IV (estomatología, oftalmología y otorrinolaringología) & 6 \\
\hline & Ginecología y Obstetricia II & 7 \\
\hline & Pediatría ll & 7 \\
\hline & Medicina Legal & 2 \\
\hline & Salud Comunitaria VIII & 2 \\
\hline \multirow{5}{*}{$\begin{array}{l}\text { 6. - curso: un mes } \\
\text { (seminarios de } \\
\text { metodología de la } \\
\text { investigación } \\
\text { biosanitaria, radiología } \\
\text { y atención primaria) }\end{array}$} & Clínica Médica & \\
\hline & Clínica Quirúrgica & \\
\hline & Pediatría y Neonatología & \\
\hline & Ginecología y Obstetricia & \\
\hline & Salud Comunitaria & \\
\hline
\end{tabular}

\section{Cronología del proyecto}

El cronograma de actuación hasta el momento ha sido el siguiente:

- Curso escolar 2012. Se impartió docencia a 30 alumnos de cuarto curso. Al final del año se evaluó el grado de satisfacción de estudiantes y pro- fesores mediante una encuesta. El resultado fue muy satisfactorio en ambos grupos. La valoración global superó el 8,5 de calificación media, sobre una puntuación máxima de 10 . Sólo hubo una valoración de 5 en una de las materias impartidas.

- Curso escolar 2013. Se impartió docencia a 52 alumnos de cuarto y a 28 de quinto curso.

- Curso escolar 2014. Se impartió docencia a 53 alumnos de cuarto, 48 de quinto y 28 de sexto curso. Diez alumnos de este último curso fueron becados para realizar un rotatorio clínico de ocho semanas de duración en los servicios de obstetricia y ginecología, pediatría, cirugía general y medicina interna de los tres hospitales universitarios de Gran Canaria (Insular, Materno Infantil y Doctor Negrín). Fueron seleccionados en función de su expediente académico, una entrevista personal y sus preferencias de especialización futura. Asimismo, se consideró favorecer la presencia de mujeres debido a la escasa representación femenina entre los estudiantes de las primeras promociones. Durante las rotaciones realizadas en Las Palmas, cada alumno desarrolló las actividades previstas de acuerdo a los objetivos de aprendizaje establecidos en el programa específico diseñado por los tutores de cada servicio. Estas rotaciones externas, además de suponer un estímulo para los estudiantes, ayudan a seleccionar a los más cualificados con la idea de que, tras su preceptiva especialización, hagan el proyecto localmente sostenible y se encarguen progresivamente de la docencia clínica en la UniZambeze.

- Curso escolar 2015. Se impartió docencia a 62 alumnos de cuarto, 50 de quinto y 35 de sexto curso. Se continuo con el plan previsto de rotaciones en hospitales de Gran Canaria de un grupo seleccionado de ocho alumnos de sexto curso.

- Curso escolar 2016. Se imparte docencia a 36 alumnos de cuarto, 63 de quinto y 54 de sexto curso. Se continúa con el plan previsto de rotaciones en hospitales de Gran Canaria de un grupo seleccionado de ocho alumnos de sexto curso.

\section{Resultados alcanzados}

Los resultados alcanzados y previstos para el futuro más inmediato se presentan en la figura 3. En 2015 se graduó la primera promoción de licenciados en medicina. Un total de 27 estudiantes egresaron y recibieron su título oficial de médico con capacidad legal para ejercer en territorio mozambiqueño. Diez de ellos han sido contratados en el Hospital Provincial de Tete. Cabe destacar que los nuevos licencia- 
dos atendieron y controlaron el brote de cólera que sufrió la provincia de Tete a principios de 2015 (https://www.msf.es/actualidad/colera-se-expande-rapidamente-mozambique).

Desde el punto de vista docente, la UniZambeze ha contratado a seis de los nuevos licenciados que trabajan en el hospital como profesores del primer ciclo de la titulación de medicina. Ellos serán, además, colaboradores locales de la ULPGC en las clases prácticas hospitalarias y en otras labores docentes y administrativas.

\section{Reflexiones finales/discusión}

Se estima que existen en torno a 2.420 facultades de medicina en todo el mundo, de las que se gradúan unos 389.000 médicos cada año. La mayor parte de estas facultades se localizan en India, China, Europa occidental e Iberoamérica y, por el contrario, se observa una llamativa escasez de centros en Asia central, Europa del este y el África subsahariana. Un total de 31 países no disponen de facultad de medicina alguna, de los cuales nueve se ubican en la región subsahariana. En 44 países sólo se dispone de un centro docente; de ellos, 17 son, una vez más, países africanos subsaharianos. Es evidente que la distribución mundial de facultades de medicina no se corresponde con la carga de enfermedad que soportan los países [4].

La educación médica en el África subsahariana experimentó su primera expansión tras finalizar el período colonizador por las potencias europeas. Antes de 1960 sólo existían cinco facultades de medicina en la región [5], pero en 1980 ya se alcanzaban los 467 centros docentes [6]. Lamentablemente, la corrupción, la hambruna y la agitación social de las siguientes décadas condujo al cierre de muchas instituciones docentes en los años ochenta y noventa.

A comienzos del siglo XXI parece existir una revitalización de la educación médica en la región, probablemente condicionada por una acusada escasez de médicos y las desigualdades en salud. Es en este contexto en el que hay que encuadrar el proyecto de cooperación que describimos en este artículo. Es un intento de reducir la brecha nortesur a través de la integración de estrategias encaminadas a conseguir la salud global en proyectos de formación de profesionales sanitarios [4], mediante la promoción de la misión social de las facultades de medicina de todo el mundo [7-9] y favoreciendo el desarrollo de estándares globales de acreditación en educación médica [10].
Figura 3. Número de estudiantes y graduados en medicina alcanzados y previstos para el futuro más inmediato.

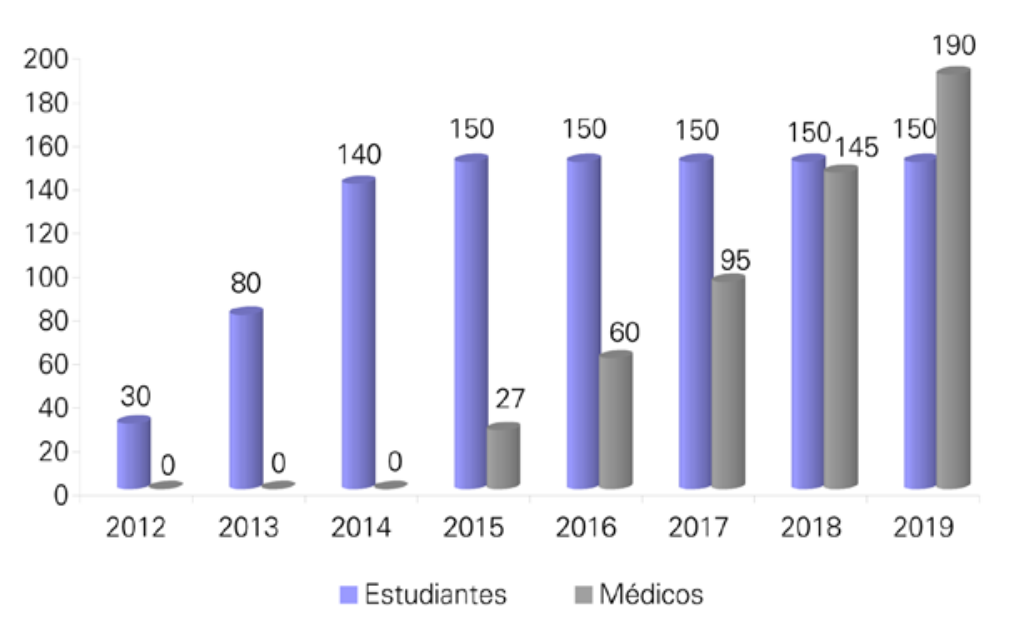

Las desigualdades en recursos humanos sanitarios son más acusadas que nunca, especialmente en África [11]. Además, se ha demostrado que la densidad de recursos humanos para la salud es un predictor de evolución favorable, especialmente en pacientes infectados por el virus de la inmunodeficiencia humana [12]. En este sentido, parece haber un consenso generalizado en la necesidad de incrementar el número de profesionales sanitarios en el África subsahariana. En función del desarrollo del proyecto descrito podemos afirmar que, en el momento de redactar este artículo, hay 60 nuevos médicos mozambiqueños. Actualmente se dispone de financiación asegurada para 2016 y 2017, lo que permite garantizar la formación de unos 200 médicos en este período (Fig. 3), la mayoría de los cuales ejercerán la profesión en su país. Es interesante señalar que está despertando un creciente interés la posibilidad de incrementar en la región, de forma paralela, el número de profesionales no médicos con experiencia clínica. Todavía no se dispone de información suficiente que permita establecer el tipo de formación que recibirían, la proporción que debería contemplarse entre los distintos profesionales sanitarios y las funciones específicas que tendría cada uno de ellos [13]. Por otra parte, es bien conocido el fenómeno de la emigración médica. Al menos uno de cada ocho médicos formados en la región subsahariana emigra a países más desarrollados [14,15], y se prevé un aumento de la escasez de médicos para los próximos años. Algunos países han perdido el $60 \%$ de sus médicos debido a la emi- 
gración como consecuencia de fenómenos de 'expulsión' (inestabilidad social, riesgos personales...) o de 'atracción' (mayores ingresos, satisfacción profesional...). Esta situación también afecta a los estudiantes, que manifiestan su intención de emigrar tan pronto les sea posible en porcentajes de hasta un $86 \%$ en algunos países [16]. No es el caso de los estudiantes de la UniZambeze. Es cierto que el derecho individual de los médicos a procurarse una vida mejor resulta innegable y no puede regularse, pero este fenómeno es fundamentalmente anglosajón y ligado a facultades que enfatizan los estándares occidentales. En todo caso, debería existir un compromiso moral de los países industrializados para no depredar a los países menos desarrollados de sus recursos humanos sanitarios.

Este proyecto ha sido y es posible gracias a los docentes que han participado de manera absolutamente voluntaria y desinteresada. En cualquier caso, es evidente que para llevarlo a cabo no basta con el voluntarismo y se precisa un soporte económico. En sus fases iniciales, este proyecto fue financiado, fundamentalmente, por las dos universidades implicadas. También colaboraron instituciones canarias, tanto gubernamentales (Cabildo Insular de Gran Canaria, Federación Canaria de Municipios), como privadas (Clínica San Roque), y organizaciones como Fundación Mapfre, Fundación Canaria Ágora y Fundación Española del Pulmón (Sociedad Española de Neumología y Cirugía Torácica). A partir de 2014, la fuente de financiación fundamental procede de la Agencia Española de Cooperación Internacional para el Desarrollo (AECID). En la convocatoria de 2013 para organizaciones no gubernamentales para el desarrollo se aprobó una subvención para cubrir los gastos de desplazamiento de los docentes en el período 2014-2016. Recientemente, la AECID ha concedido una nueva financiación, en su convocatoria de 2015 de proyectos de innovación para el desarrollo, para el período 20162017. Con el presupuesto concedido sólo se habría podido financiar la estancia de tres médicos en Tete por un período de cuatro años, lo que contrasta con la formación de cerca de 100 médicos desde que comenzó este proyecto. En todo caso, aunque contar con un capital para impulsar un proyecto es algo complejo, mantener una fuente de financiación que permita su sostenibilidad resulta todavía más complicado, especialmente cuando se alcanzan los diez años de desarrollo [17]. Por ello, uno de los objeti- vos es ir formando, también desde el punto de vista docente, a los médicos egresados más destacados para que se hagan cargo de manera progresiva de la formación clínica de generaciones futuras de médicos en la UniZambeze. Además, en los próximos años se pretende impulsar un sistema de teleformación interactiva que disminuya los desplazamientos de profesores desde Las Palmas y facilite la sostenibilidad del proyecto. Uno de los problemas para impulsar este sistema de teleformación son las malas infraestructuras locales en lo que respecta a la conexión por Internet.

\section{Bibliografía}

1. Gukas ID. Global paradigm shift in medical education: issues of concern for Africa. Med Teach 2007; 29: 887-92.

2. World Health Organization. The world health report: working together for health. Geneva: WHO; 2006.

3. Mullan F, Panosian C, Cuff P. Healers abroad: Americans responding to the human resource crisis in HIV/AIDS Washington: National Academy Press; 2005.

4. Frenk J, Chen L, Bhutta ZA, Cohen J, Crisp N, Evans T, et al. Health professionals for a new century: transforming education to strengthen health systems in an interdependent world. Lancet 2010; 376: 1923-58.

5. Mullan F, Frehywot S, Omaswa F, Buch E, Chen C, Greysen SR, et al. Medical schools in sub-Saharan Africa. Lancet 2011; 377: 1113-21.

6. World Health Organization. WHO Avicenna World Directory of Medical Schools. URL: http://avicenna.ku.dk/database/ WHO directory/. [01.06.2016].

7. Van Niekerk JP. Missions of a medical school: an African perspective. Acad Med 1999; 8 (Suppl): 38-44.

8. Sewankambo N. Academic medicine and global health responsibilities. BMJ 2004; 329: 752-3.

9. Boelen C. Social accountability and accreditation: a new frontier for educational institutions. Pedagogie Medicale 2008; 9: 235-44.

10. Burdick W. Challenges and issues in health professions education in Africa. Med Teach 2007; 29: 882-6.

11. Human Resources for Health. Overcoming the crisis. Joint Learning Initiative. Cambridge: Harvard University; 2004 URL: http://www.hrhresourcecenter.org/node/31. [01.06.2016].

12. Kober K, Van Damme W. Scaling up access to antiretroviral treatment in southern Africa: who will do the job? Lancet 2004, 364: 103-7.

13. Dovlo D. Using mid-level cadres as substitutes for internationally mobile health professionals in Africa. A desk review. Hum Resour Health 2004; 2: 7.

14. Mullan F. The metrics of the physician brain drain. N Engl J Med 2005; 353: 1810-8.

15. Hagopian A, Thompson MJ, Fordyce M, Johnson KE, Hart LG. The migration of physicians from sub-Saharan Africa to the United States of America: measures of the African brain drain. Hum Resour Health 2004; 2: 17.

16. Awases M, Gbary A, Nyoni J, Chatora R. Migration of health professionals in six countries: a synthesis report. Brazzaville: WHO Regional Office for Africa; 2004. URL: http://www. hrhresourcecenter.org/node/61. [01.06.2016].

17. Broadhead RL, Muula AS. Creating a medical school for Malawi: problems and achievements. BMJ 2002; 325: 384-7. 